\title{
No difference in analgesic effects between non-steroidal anti-inflammatory drugs and acetaminophen at the latest two weeks after injury or surgery
}

\author{
Katsuhiro Toda* \\ Department of Rehabilitation, Fukuyama Rehabilitation Hospital, 15-41, 2-choume, Myoujin-chou, Fukuyama city, Hiroshima, 721-0961, Japan
}

\begin{abstract}
Introduction: An appropriate administration period for non-steroidal anti-inflammatory drugs (NSAIDs) after the occurrence of nociceptive pain has not yet been established.

Study 1: Methods: NSAIDs were changed to acetaminophen in 59 patients (42 women) who were regularly administered NSAIDs on admission. The average age of patients was 76.0 years old (31-96). The average period from admission to the discontinuation of NSAIDs was 1.5 days (0-10). The average period from injury, surgery, or the occurrence of pain to the discontinuation of NSAIDs was 25.7 days (3-142).

Results: Eleven patients (18.6\%) showed improvements, $45(76.3 \%)$ no change, and $3(5.1 \%)$ exacerbation. The term from injury, surgery, or the occurrence of pain to the administration of acetaminophen was 3 days (1 patient), 7 (1), 8 (3), 9 (2), 10 (2), 11 (1), 12 (5), 13 (5), 14 (2), 15 (2), 17 (2), 18 (2), 19 (2), 20 (1), 21 (2), $22-28$ (8), 29-35 (7), 36-42 (4), 43 (1), 50 (1), 58 (1), 63 (1), 76 (1), 77 (1), and 142 (1).
\end{abstract}

Study 2: Methods: Among the 59 patients eligible for Study 1,54 (38 women) with an average age of 76.3 years (38-96) for whom acetaminophen was discontinued were included in Study 2. The mean administration period of acetaminophen was 8.8 days (4-35).

Results: Nine patients (16.7\%) showed improvements, 40 (74.1\%) no change, 1 (1.9\%) exacerbation, and 4 (7.4\%) unknown.

Conclusions: The significance of medication with NSAIDs typically disappears at the latest two weeks after injury or surgery.

\section{Introduction}

Non-steroidal anti-inflammatory drugs (NSAIDs) and acetaminophen are effective for nociceptive pain. The analgesic effects of NSAIDs are generally stronger than those of acetaminophen, while acetaminophen has fewer adverse effects. NSAIDs are often prescribed after injury or surgery. Once pain is relieved, NSAIDs are typically changed to acetaminophen or discontinued.

Patients who have been injured, undergone surgery, had strokes, or have other conditions are referred from acute hospitals to Fukuyama Rehabilitation Hospital. Many patients are prescribed NSAIDs on admission. An appropriate administration period for NSAIDs has not yet been established. In the present study, NSAIDs were promptly changed to acetaminophen in patients administered NSAIDs on admission and changes in pain were examined. Acetaminophen was subsequently discontinued and changes in pain were examined.

This is a retrospective study that reported the outcomes of the routine treatment employed. The Ethical Review Board of Fukuyama Rehabilitation Hospital approved this study.

\section{Study 1 (Figure 1, Table 1) \\ Patients and methods}

Fifty-nine patients who were regularly administered NSAIDs on admission to Fukuyama Rehabilitation Hospital between January 2014 and October 2016 and whose NSAIDs were changed to acetaminophen were included in this study. NSAIDs were prescribed by acute hospitals. Changes in pain were observed after NSAIDs were changed to acetaminophen. Patients were verbally asked about changes in pain, which were classified as improvements, no change, exacerbation, and unknown; unknown was regarded as no change. The following cases were excluded from the present study. I: Patients who did not wish to change from NSAIDs to acetaminophen and NSAIDs were suddenly discontinued. II: NSAIDs were not discontinued at the request of patients. III: Weak opioids were administered for severe pain. IV: NSAIDs were administered over the long term for diseases that did not require admission such as low back pain. V: Changes in pain were not assessed due to severe dementia. The following explanation was given to patients, and oral informed consent was obtained: "May I change the current analgesic to a new analgesic that is slightly weaker than the current analgesic, but with less adverse effects?" Anti-ulcerative drugs in combination with NSAIDs were discontinued and acetaminophen was administered without anti-ulcerative drugs. The 59 patients enrolled

${ }^{*}$ Correspondence to: Katsuhiro Toda, Department of Rehabilitation, Fukuyama Rehabilitation Hospital, 15-41, 2-choume, Myoujin-chou, Fukuyama city, Hiroshima, 721-0961, Japan, Tel: +81-84-916-5500; FAX: +81-84-916-5511; E-mail: goutattack@yahoo.co.jp

Key words: non-steroidal anti-inflammatory drugs, acetaminophen, nociceptive pain

Received: May 18, 2018; Accepted: May 25, 2018; Published: May 31, 2018 


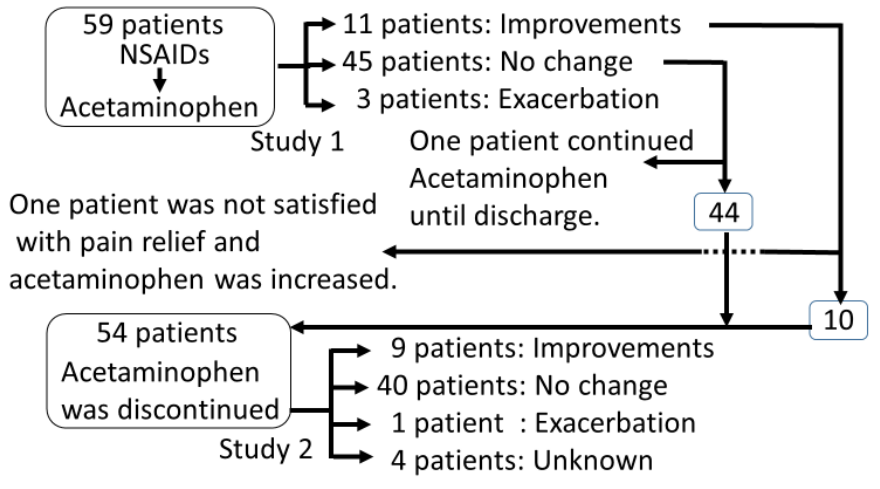

Figure 1. Algorithm of Study 1 and 2

Table 1. Study 1 results

\begin{tabular}{|c|c|c|}
\hline Study 1 & & \\
\hline Female/Male & $42 / 17$ & \\
\hline Age (year) & $76.0 \pm 14.0(31-96)$ & \\
\hline $\begin{array}{l}\text { Pain occurrence- } \\
\text { Discontinuation of NSAIDs } \\
\text { (day) }\end{array}$ & $25.7 \pm 22.1(3-142)$ & \\
\hline $\begin{array}{l}\text { Admission-Discontinuation } \\
\text { of NSAIDs (day) }\end{array}$ & $1.5 \pm 2.0(0-10)$ & \\
\hline \multirow[t]{6}{*}{ NSAIDs } & $200 \mathrm{mg}$ celecoxib & 31 \\
\hline & $180 \mathrm{mg}$ loxoprofen & 17 \\
\hline & $120 \mathrm{mg}$ loxoprofen & 8 \\
\hline & $400 \mathrm{mg}$ indometacin farnesyl & 1 \\
\hline & $200 \mathrm{mg}$ sulindac & 1 \\
\hline & $12 \mathrm{mg}$ lornoxicam & 1 \\
\hline \multirow[t]{4}{*}{ Acetaminophen $(200 \mathrm{mg})$} & 6 tablets & 2 \\
\hline & 4 tablets & 1 \\
\hline & 3 tablets & 29 \\
\hline & 2 tablets & 27 \\
\hline \multirow[t]{4}{*}{ Results } & Improvements & $11(18.6 \%)$ \\
\hline & No change & $45(76.3 \%)$ \\
\hline & Exacerbation & $3(5.1 \%)$ \\
\hline & Unknown & 0 \\
\hline
\end{tabular}

consisted of 42 women and 17 men with an average age of $76.0 \pm 14.0$ years (range: 31 to 96 years old). The average period from admission to the discontinuation of NSAIDs (change to acetaminophen) was $1.5 \pm$ 2.0 days (range: 0 to 10 days). The average period from injury, surgery, or the occurrence of pain to the discontinuation of NSAIDs (change to acetaminophen) was $25.7 \pm 22.1$ days (range: 3 to 142 days). Thirty-one patients received 2 tablets $(100 \mathrm{mg})$ of celecoxib, 17 patients 3 tablets $(60$ $\mathrm{mg}$ ) of loxoprofen, 8 patients 2 tablets $(60 \mathrm{mg}$ ) of loxoprofen, 1 patient 2 capsules $(200 \mathrm{mg})$ of indometacin farnesyl, 1 patient 2 capsules (100 $\mathrm{mg}$ ) of sulindac, and 1 patient 3 tablets $(4 \mathrm{mg})$ of lornoxicam. Two patients received 6 tablets $(200 \mathrm{mg})$ of acetaminophen, 1 patient 4 tablets, 29 patients 3 tablets, and 27 patients 2 tablets. Patients who received 2 tablets (capsules) of NSAIDs were typically administered 2 or 4 tablets of acetaminophen, while those who received 3 tablets generally received 3 or 6 tablets of acetaminophen.

\section{Results}

Pain was relieved in 11 patients (18.6\%), remained unchanged in 45 (76.3\%), worsened in $3(5.1 \%)$, and was unknown in 0 (Table 1 ).

Of the three patients whose pain worsened, pain in the lower extremities of an ambulatory 31-year-old man with spinal cord injury worsened slightly four days after the change to acetaminophen.
However, he wished to continue acetaminophen because the degree of the deterioration was mild. Pain worsened two days after the change to acetaminophen in a 78-year-old female, and, thus, celecoxib, the NSAIDs on admission, was administered again. She had strictly complied with her previous medication regimen, but then occasionally did not take celecoxib, when the pain did not change. An 88-year-old female provided an ambiguous response to the question asked two days after the change to acetaminophen. She finally stated that her pain worsened, and acetaminophen was changed to loxoprofen, the NSAIDs on admission. Loxoprofen was changed to celecoxib four days later, which was changed to acetaminophen after 15 days. Acetaminophen was discontinued 20 days after its administration and pain remained unchanged. These three patients received acetaminophen 22 days after surgery, 36 days after injury, and 136 days after surgery.

The term from injury, surgery, or the occurrence of pain to the administration of acetaminophen was 3 days (1 patient), 7 (1), 8 (3), 9 (2), 10 (2), 11 (1), 12 (5), 13 (5), 14 (2), 15 (2), 17 (2), 18 (2), 19 (2), 20 (1), 21 (2), 22-28 (8), 29-35 (7), 36-42 (4), 43 (1), 50 (1), 58 (1), 63 (1), 76 (1), 77 (1), and $142(1)$.

Forty-three patients had fractures in and/or underwent surgery on the extremities and/or pelvis (femoral head replacement 12, $\gamma$-nail 7, screw fixation of a femoral neck fracture 4), 11 fractures in and/or surgery on the vertebral column, 1 spinal cord injury, 1 neck pain, 1 postherpetic neuralgia, 1 aortic vascular prosthesis replacement surgery, and 1 above-knee amputation.

\section{Study 2 (Figure 1, Table 2)}

\section{Patients and methods}

Among the 59 patients eligible for Study 1,54 excluding 3 whose pain worsened, 1 who was not satisfied with pain relief and acetaminophen was increased, and 1 who continued acetaminophen until discharge as requested by the patient, were included in Study 2. Pain was relieved in 10 patients and remained unchanged in 44. Acetaminophen was discontinued approximately one week after administration and changes in pain were examined in 54 patients using the same method as that in Study 1 . The mean administration period of acetaminophen was $8.8 \pm$ 6.3 days (range: 4 to 35 days). Fifty-four patients consisted of 38 women and 16 men with an average age of $76.3 \pm 13.2$ years (range: 38 to 96 years old). One patient received 6 tablets $(200 \mathrm{mg})$ of acetaminophen, 27 patients 3 tablets, and 26 patients 2 tablets.

\section{Results}

Of the 54 patients for whom NSAIDs were changed to acetaminophen, which was subsequently discontinued, pain was relieved in 9 patients $(16.7 \%)$, remained unchanged in $40(74.1 \%)$, worsened in 1 (1.9\%), and was impossible to evaluate in 4 (7.4\%) (Table 2).

Table 2. Study 2 results
\begin{tabular}{|l|l|l|}
\hline Study 2 & & \\
\hline Female/Male & $38 / 16$ & \\
\hline Age (year) & $76.3 \pm 13.2(38-96)$ & \\
\hline $\begin{array}{l}\text { Acetaminophen } \\
\text { administration (day) }\end{array}$ & $8.8 \pm 6.3(4-35)$ & \\
\hline Acetaminophen (200 mg) & 6 tablets & 1 \\
\hline & 3 tablets & 27 \\
\hline & 2 tablets & 26 \\
\hline Results & Improvements & $9(16.7 \%)$ \\
\hline & No change & $40(74.1 \%)$ \\
\hline & Exacerbation & $1(1.9 \%)$ \\
\hline & Unknown & $4(7.4 \%)$ \\
\hline
\end{tabular}


Of the nine patients whose pain decreased after the discontinuation of acetaminophen, the term from discontinuation to the worsening of pain was 0 days in 1 patient, 1 day in 5,2 days in 1,8 days in 1 , and 10 days in 1 .

\section{Discussion}

NSAIDs and acetaminophen are effective for nociceptive pain. The analgesic effects of NSAIDs are generally stronger than those of acetaminophen, while acetaminophen has fewer adverse effects. Therefore, if no difference exists in analgesic effects between these two medicines, acetaminophen is recommended for administration. In patients for whom pain does not change after the switch from NSAIDs to acetaminophen, both medicines may be equally effective or ineffective. Some patients stated that analgesic medicine was not needed because pain levels were mild. Thus, acetaminophen should be administered instead of NSAIDs, when the pain remains unchanged. Moreover, acetaminophen may not exert analgesic effects. If NSAIDs are discontinued after admission, the suggestive effect (the inverseplacebo effect) of discontinuing analgesic medicine may exacerbate pain. Therefore, in order to reduce the suggestive effect (the inverseplacebo effect), NSAIDs were not suddenly discontinued and were changed to acetaminophen.

The adverse effects of NSAIDs include renal, cardiac, and gastrointestinal tract disturbances. Gastrointestinal tract disturbances are the most problematic adverse effects of NSAIDs for the following reasons: I: high incidence, II: may be fatal, III: patients with NSAIDs ulcers often do not have subjective symptoms, IV: there is currently no method to detect early asymptomatic NSAIDs ulcers, and V: NSAIDs ulcers sometimes develop within approximately one week [1]. At least 16,500 NSAIDs-related deaths due to gastrointestinal events are estimated to occur each year among arthritis patients alone in the U.S [2]. NSAIDs have also been showing to exacerbate renal disturbances. This study included a patient with moderate renal disturbance with contraindications to NSAIDs.

Pain after injury or surgery is typically nociceptive pain. According to the central sensitization theory $[3,4]$, which is gaining recognition, whatever the reason for pain, if all types of pain persist, the central nervous system such as the brain is sensitized. Sensitization of the central nervous system itself causes pain, resulting in central neuropathic pain. Thus, the persistence of nociceptive pain results in a combined pain of nociceptive and neuropathic pain. If neuropathic pain patients undergo surgery or injury, they have the combined pain of nociceptive and neuropathic pain. Some patients received analgesic medicine for neuropathic pain after the completion of the present study.

There are two mechanisms of action by analgesic medicine. The first mechanism involves analgesic effects while maintaining a constant blood concentration of the medicine, which is regarded as a true analgesic effect. The other mechanism includes the suggestive effect, natural course, and coincidence, which are collectively known as the placebo effect. There are also two mechanisms responsible for the aggravation of pain after analgesic medicine is discontinued or changed. One mechanism involves the disappearance of the analgesic effects of analgesic medicine and differences in analgesic effects between two analgesic medicines. The other mechanism involves the suggestive effect, natural course, and coincidence. Even if medicine that has no analgesic effect is discontinued, pain is sometimes exacerbated. This is herein referred to as the inverse-placebo effect. The causes of disagreements between pharmacological analgesic effects and clinical analgesic effects include individual differences in responses to medicine, the placebo effect, and the inverse-placebo effect.
In study 1 , pain was relieved in 11 out of 59 patients whose NSAIDs were changed to acetaminophen. However, this result does not indicate that the analgesic effects of acetaminophen were stronger than those of NSAIDs in these patients. The analgesic effects of NSAIDs are generally stronger than those of acetaminophen. I estimate that it is due to the placebo effect or the natural course. In study 1, pain worsened in 3 of the 59 patients whose NSAIDs were changed to acetaminophen. In one patient (31-year-old man), the reason may have been due to the difference in analgesic effects between NSAIDs and acetaminophen. Because one patient (78-year-old female) had strictly complied with her previous medication regimen, an inverse-placebo effect cannot be excluded. Expression of pain in the remaining one patient (88-year-old female) was ambiguous and pain did not change after NSAIDs were changed to acetaminophen again. Therefore, it was unknown whether there was a difference in analgesic effects between NSAIDs and acetaminophen on admission. Only 1 of the 59 patients showed a definite difference in analgesic effects between NSAIDs and acetaminophen on admission. In study 2 , acetaminophen may have had no analgesic effects in two of the nine patients, because the pain became exacerbated on Days 8 and 10 after discontinuation of administration.

The period from injury, surgery, or the occurrence of pain to the discontinuation of NSAIDs (change to acetaminophen) was within 14 days in 22 patients (37.3\%) in study 1 . The periods from injury or surgery to the discontinuation of NSAIDs (change to acetaminophen) were 22 days, 36 days, and 136 days in three patients whose pain worsened after the change from NSAIDs to acetaminophen. Pain did not worsen in any patients whose period from injury, surgery, or the occurrence of pain to the discontinuation of NSAIDs (change to acetaminophen) was between 7 and 14 days. Thus, the significance of NSAIDs two weeks after injury, surgery, or the occurrence of pain appears to be negligible. NSAIDs may be changed to acetaminophen or discontinued at the latest two weeks after injury or surgery. The longterm administration of NSAIDs without the confirmation of analgesic effects is undesirable. Based on the results of the present study, NSAIDs need to be changed to acetaminophen or discontinued at the latest two weeks after injury or surgery. If pain is severe, weak opioids are recommended, whereas if neuropathic pain is suspected two weeks after injury or surgery, analgesic medicine for neuropathic pain needs to be administered. The significance of the administration of NSAIDs at the latest two weeks after injury or surgery needs to be confirmed. Although shortening of this period of two weeks may be possible, the results of this study cannot confirm it.

NSAIDs alone must not be administered in the following three cases. I: When pain is mild, NSAIDs need to be changed to topical medicine or acetaminophen. II: When pain is severe, such as spinal compression fractures, weak opioids need to be substituted for NSAIDs or administered in combination with NSAIDs. III: Analgesic medicine such as Neurotropin ${ }^{\circledR}$ (an extract from the inflamed skin of vaccinia virus-inoculated rabbits) and pregabalin need to be administered for neuropathic pain. When neuropathic pain is suspected, but not confirmed, strong analgesic medicine is recommended such as diclofenac sodium suppositories. If suppository NSAIDs with potent analgesic effects are ineffective, oral NSAIDs should not be administered and I administer analgesics for neuropathic pain or weak opioids.

\section{Conclusion}

Of the 59 patients who were regularly administered NSAIDs on admission and whose NSAIDs were changed to acetaminophen, pain was relieved in 11 patients (18.6\%), remained unchanged in $45(76.3 \%)$, and worsened in 3 (5.1\%). Of the 54 patients for whom NSAIDs were changed to acetaminophen, which was then discontinued, pain was 
relieved in 9 patients (16.7\%), remained unchanged in $40(74.1 \%)$, worsened in $1(1.9 \%)$, and was impossible to evaluate in $4(7.4 \%)$. The significance of NSAIDs generally appears to disappear at the latest two weeks after injury or surgery.

\section{Disclosure and conflicts of interest}

No conflicts of interest

\section{Acknowledgements}

I thank Medical English Service for reviewing this manuscript.

\section{References}

1. Machado GC, Maher CG, Ferreira PH, Day RO, Pinheiro MB, et al. (2017) Nonsteroidal anti-inflammatory drugs for spinal pain: a systematic review and metaanalysis. Ann Rheum Dis. 76: 1269-1278. [Crossref]

2. Singh G (1998) Recent considerations in nonsteroidal anti-inflammatory drug gastropathy. Am J Med. 105: 31S-38S. [Crossref]

3. Clauw DJ (2015) Diagnosing and treating chronic musculoskeletal pain based on the underlying mechanism(s). Best Pract Res Clin Rheumatol. 29: 6-19. [Crossref]

4. Schaible HG (2012) Mechanisms of Chronic Pain in Osteoarthritis. Curr Rheumatol Rep. 14: 549-556.

Copyright: (C2018 Toda K. This is an open-access article distributed under the terms of the Creative Commons Attribution License, which permits unrestricted use, distribution, and reproduction in any medium, provided the original author and source are credited. 